\title{
Effects of particle size of dielectric fillers on the output performance of piezoelectric and triboelectric nanogenerators
}

\author{
Xiao MENG ${ }^{a}$, Zhuo ZHANG ${ }^{a}$, Dabin $\mathrm{LIN}^{a, *}$, Weiguo LIU ${ }^{a}$, Shun ZHOU ${ }^{a}$, \\ Shaobo $\mathrm{GE}^{a}$, Yongming $\mathrm{SU}^{a}$, Chang $\mathrm{PENG}^{b}$, Lin $\mathrm{ZHANG}^{c, *}$ \\ ${ }^{a}$ School of Opto-electronical Engineering, Xi'an Technological University, Xi'an 710021, China \\ ${ }^{b}$ Department of Mechanical and Aerospace Engineering, North Carolina State University, Raleigh, NC 27695, USA \\ ${ }^{c}$ Electronic Materials Research Laboratory, Key Laboratory of the Ministry of Education \& International \\ Center for Dielectric Research, School of Electronic Science and Engineering, Faculty of \\ Electronic and Information Engineering, Xi'an Jiaotong University, Xi'an 710049, China
}

Received: January 26, 2021; Revised: March 31, 2021; Accepted: April 9, 2021

(C) The Author(s) 2021.

\begin{abstract}
Recently, piezoelectric/triboelectric nanogenerators based on piezoelectric composite materials have been intensively studied to achieve high electrical output performance. In this work, flexible $\mathrm{BaTiO}_{3}$ (BT)/PDMS nanocomposite films with various sizes and concentrations were fabricated and used as the nanogenerators. The influence of dielectric properties on the electrical output of nanogenerators was studied as well as the structure of the composites. The dielectric constant increased from 6.5 to 8 with the concentration of BT nanoparticles and decreased with the frequency from $10^{2}$ to $10^{6} \mathrm{~Hz}$. Furthermore, the dielectric constant showed $11 \%$ decrease with the temperature range from 30 to $180{ }^{\circ} \mathrm{C}$. It was found that the concentration of BT nanoparticles has promoted the electrical output of nanogenerators. The output voltage and current are all enhanced with the BT nanoparticles, which reached $200 \mathrm{~V}$ and $0.24 \mu \mathrm{A}$ in TENG with $40 \mathrm{wt} \% \mathrm{BT}$ nanoparticles, respectively. The selected device exhibited the power of $0.16 \mathrm{~mW}$ and employed to demonstrate its ability to power wearable/portable electronics by lighting the LEDs.
\end{abstract}

Keywords: polymer-matrix composites; nano composites; smart materials; electrical properties

\section{Introduction}

Generating electricity with an effective and environmental way is a hot spot in the current energy field. There are many types of wasted mechanical energy in our daily life and nature, including human motion (shaking hands, walking, running) [1-3], vibration [4,5], wind

\footnotetext{
* Corresponding authors.

E-mail: D. Lin, dabinlin@xatu.edu.cn;

L. Zhang, zhanglin.materials@gmail.com
}

[6], waves [7], falling water [8], and sound [9]. Up to now, a variety of mechanical energy harvesters, such as electrostatic, electromagnetic, piezoelectric, and triboelectric devices have been invented to collect wasted mechanical energy and convert to the electrical energy [10-12]. With the rapid development of modern technology, nanotechnology and nanostructured materials have been involved in design and fabrication of energy harvesters. Compared with other generators [13,14], piezoelectric nanogenerators (PENG) [15] and triboelectric nanogenerators (TENG) [16] are more portable and 
flexible which can be utilized in various applications. For TENG, there are four different modes, contact separation mode [17], lateral sliding mode [18], single electrode mode [19], freestanding triboelectric layer mode [20], which can suit for various applications.

Until now, many researchers focused on improving the output performance of PENG and TENG with a compact structure, a high sensitivity, and a long durability for self-powered sensors [21-25]. To make the nanogenerators suit for more applications, polymer/ elastomer-based nanocomposites which is a polymer matrix reinforced with nanoparticles have been intensively investigated because of their merits of excellent flexibility/stretchability and easy processing. For example, PDMS [26], PMMA [27], and PVDF [28-31] are mainly used as the matrix. Among them, PDMS as a biocompatible elastomer has advantages of flexible, transparent, and simple fabrication, which has been widely used in flexible nanogenerators [32-35]. For polymer/elastomer-based PENG and TENG, there are two effective methods to improve the output performance. In one hand, the working areas of the devices have the strong impact on the output performance. To improve output power, nanostructures are fabricated on the surface of the contact layers to increase the working area [36]. In another hand, selecting the inorganic fillers with high dielectric constant which can provide an enhanced charge storage for the devices [37]. For the selection of fillers, a variety of materials have been used to fabricate nanogenerators such as PZT [38], ZnO [39,40], and $\mathrm{BaTiO}_{3}$ (BT) [41,42]. However, the toxicity of PZT and the low output performance of $\mathrm{ZnO}$ limited their applications. $\mathrm{BaTiO}_{3}$ exhibited a high dielectric constant, stable properties over wide temperature range, and controlled size and shape with simple fabrication, which has been widely used in different flexible electronics [43-49].

In this work, we focused on investigating the dielectric properties of BT/PDMS with various particle sizes and output performance of nanogenerators based on the composite films. Commercially available $\mathrm{BaTiO}_{3}$ nanoparticles with uniform size and shape distributions are selected as the filler to guarantee the expected experimental design. PDMS-based nanocomposites with different weights of BT nanoparticles were fabricated, and the different concentrations of nanocomposites were used in PENG and TENG devices. The phase and morphology of the nanocomposites were characterized by X-ray diffraction and scanning electron microscope. In addition, the temperature and frequency dependence of dielectric constant and loss was studied, respectively. Finally, the open-circuit voltage and short-circuit current were measured to show the output performance of the PENG and TENG. To demonstrate the practical and commercial applications, the power generated by the TENG device was utilized to power up various low-power wearable/portable electronics.

\section{Experimental procedures}

\section{1 Materials}

$\mathrm{BaTiO}_{3}$ nanoparticles $(99.9 \%$, diameter of $\sim 30 \mathrm{~nm}$ ) were purchased from the Alfa Aesar, USA. The PDMS (Sylgard ${ }^{\circledR} 184$ silicone elastomer base and silicone elastomer curing agent) was purchased from the Dow Corning Corporation, USA. Indium tin oxide coated PET (surface resistively $60 \Omega / \mathrm{sq}, 1 \mathrm{ft} \times 1 \mathrm{ft} \times 5 \mathrm{mil}$ ) was purchased from ALDRICH. $\mathrm{Bi}_{2} \mathrm{O}_{3}, \mathrm{TiO}_{2}$, and $\mathrm{BaCO}_{3}$ were used as raw materials and employed by Alpha Chemicals. The $\mathrm{NaCl}$ and $\mathrm{KCl}$ were purchased from Sinopharm Chemical Reagent Co Ltd. The copper tape and Indium tin oxide coated PET were bought from Sigma-Aldrich, Co., Ltd., USA.

\section{2 Synthesis of micro-scale $\mathrm{BaTiO}_{3}$ flake}

The micro-scale BT flake was synthesized by the combination of the molten salt technique and topological synthesis method, and the process was divided into three steps. Firstly, the $\mathrm{Bi}_{4} \mathrm{Ti}_{3} \mathrm{O}_{12}$ platelet was synthesized by a molten salt method. Secondly, $\mathrm{BaBi}_{4} \mathrm{Ti}_{4} \mathrm{O}_{15}$ was synthesized through a molten salt method by using reactants of $\mathrm{BaBi}_{4} \mathrm{Ti}_{4} \mathrm{O}_{12}$, and some of $\mathrm{Bi}^{3+}$ converted into $\mathrm{Ba}^{2+}$. Thirdly, $\mathrm{BT}$ platelets were obtained by a topochemical reaction between $\mathrm{BaBi}_{4} \mathrm{Ti}_{4} \mathrm{O}_{15}$ and $\mathrm{BaCO}_{3}$.

\section{3 Preparation of BT/PDMS films for PENG and TENG devices}

Figure 1 shows the fabrication process of BT/PDMS films and PENG and TENG devices. The silicone elastomer base and silicone elastomer curing agent were put into a beaker with the ratio of 10:1, then the solution was mixed thoroughly, and bubbles was degassed. Next, the solution with substrate was placed on the spin coating machine with a speed of $800 \mathrm{rpm}$ for $30 \mathrm{~s}$ to get a uniform PDMS film, and then cured it 
at $80{ }^{\circ} \mathrm{C}$ for $2 \mathrm{~h}$. The two solutions of PDMS were put into a beaker with a ratio of 10:1, and then the BT nanoparticles were dispersed into the PDMS at various concentrations of $5,10,20,30$, and $40 \mathrm{wt} \%$, respectively. The following steps are the same as the fabrication of pure PDMS film. Finally, different BT/PDMS films with uniform filler distribution and excellent flexibility can be obtained, as shown in Figs. 2(a) and 2(b).

For the PENG, the different concentrations of $\mathrm{BT} /$
PDMS composite film acted as the dielectric film. The copper tape was attached to both surfaces of the film, as shown in Fig. 2(c). For the TENG, the composite film was placed into the two commercial ITO-PET films. And then four spacers which built a gap between the composite film and ITO-PET were put on the four corners of the composite film. The $\mathrm{Cu}$ tapes were attached to the ITO layer to connect the load, as shown in Fig. 2(d).

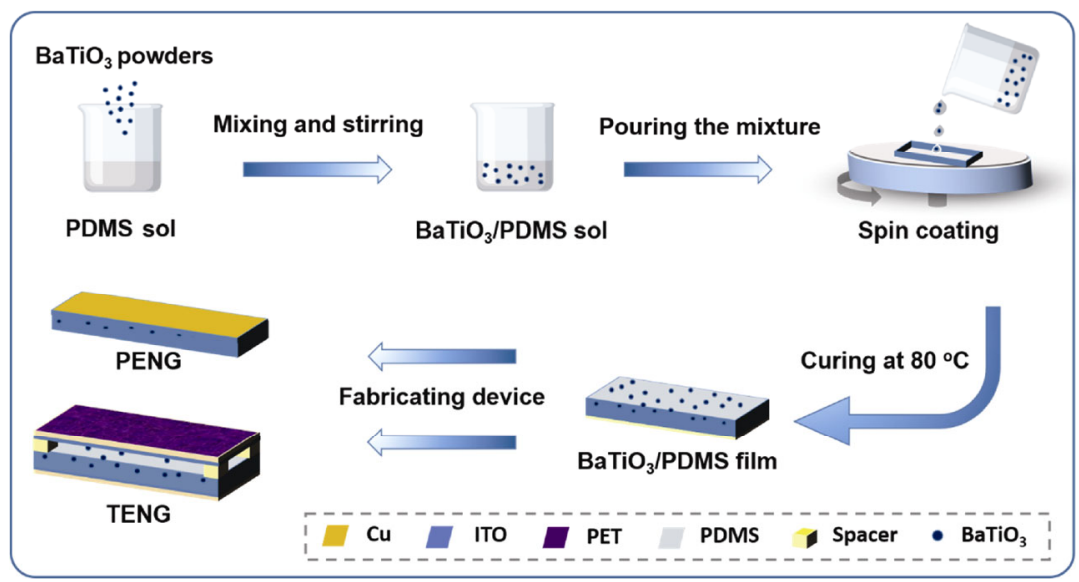

Fig. 1 Schematic of preparation of $\mathrm{BaTiO}_{3} / \mathrm{PDMS}$ nanocomposite for PENG and TENG devices: mixing $\mathrm{BaTiO}_{3}$ powders with PDMS solution, casting and spin coating the mixture, curing the mixture at high temperature, and adding electrodes for PENG and TENG devices.
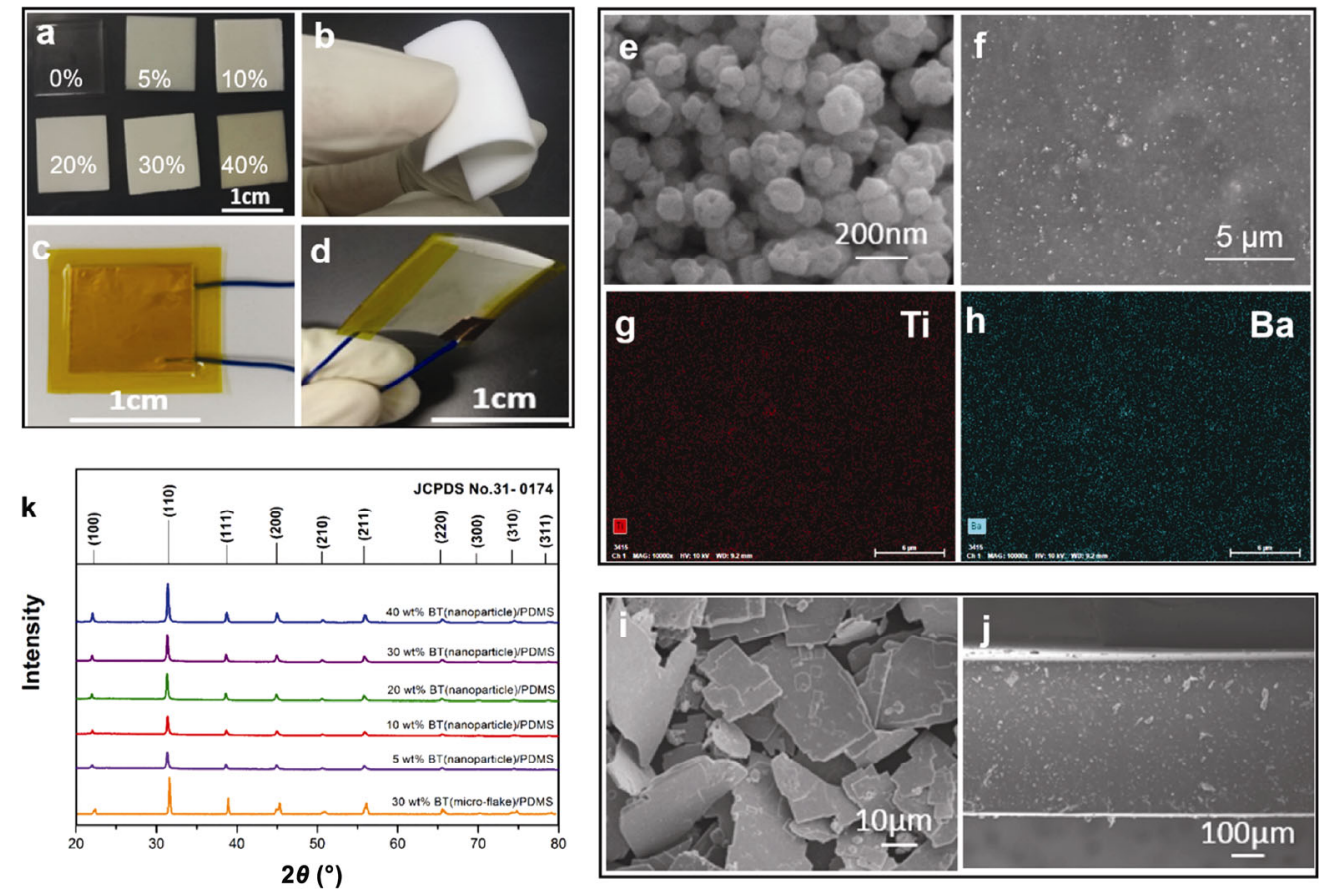

Fig. 2 Images of films and devices: (a) a digital photograph of BT/PDMS nanocomposites with different BT contents from 0\% to $40 \%$, (b) a digital photograph of flexible nanocomposite film, and images of (c) the PENG and (d) the TENG devices. Morphologies from SEM images: (e) the $\mathrm{BaTiO}_{3}$ nanoparticles, (f) the cross-section of BT/PDMS composite film with 40 wt $\%$ of BT nanoparticles. (g, h) EDS mapping of film with uniform distribution of Ti and Ba in the PDMS, (i) the SEM image of $\mathrm{BaTiO}_{3}$ flakes, (j) the cross-sectional SEM image of BT/PDMS composite film with $30 \mathrm{wt} \%$ of BT flakes. Structure of all samples: (k) the X-ray diffraction spectra of various composite films and the XRD standard card of $\mathrm{BaTiO}_{3}$. 


\section{4 Characterization and measurements}

The morphologies of the composite surfaces were studied by field emission scanning electron microscopy (Phenom LE). The X-ray diffraction (XRD) analysis was carried out by using an Ultima IV machine. The dielectric constant and loss were measured with a Precision LCR Meter (E4980AL). The open-circuit voltage $\left(V_{\mathrm{OC}}\right)$ and short-circuit current $\left(I_{\mathrm{SC}}\right)$ of all devices were measured with a mixed domain oscillometer (MDO3022), an electrometer (KEITHLEY 6517A), and a laptop for data collection.

\section{Results and discussion}

\section{1 Structure, morphology, and flexibility}

Figure 2(e) shows the high magnified SEM images of morphologies of BT nanoparticles; the shape of BT nanoparticles is spherical, and the average size is about 50-80 nm. To know the detailed morphologies of composite film, the cross-sectional SEM images of nanocomposite with $40 \mathrm{wt} \%$ of BT are exhibited in Fig. 2(f), which reveals the uniform distribution of BT nanoparticles. the EDX mapping of elements Ti and Ba in the same observation area is shown in Figs. 2(g) and 2(h) to clearly prove the homogeneous microstructure. Figure 2(i) shows the morphologies of the micro-sized $\mathrm{BaTiO}_{3}$ flakes with the size of $20 \mu \mathrm{m} \times 40 \mu \mathrm{m}$. Figure $2(\mathrm{j})$ is the cross-sectional SEM image of the composite film with $30 \mathrm{wt} \%$ of BT flakes. The microstructure of BT flakes in the PDMS matrix is shown in the cross-sectional SEM image and the thickness of the composite film is about $450 \mu \mathrm{m}$. Figure $2(\mathrm{k})$ shows the XRD characteristic of the composite films with different $\mathrm{BaTiO}_{3}$ with 5, 10, 20, 30, $40 \mathrm{wt} \%$ and with $30 \mathrm{wt} \%$ of BT flakes in the range from $20^{\circ}$ to $80^{\circ}$. The

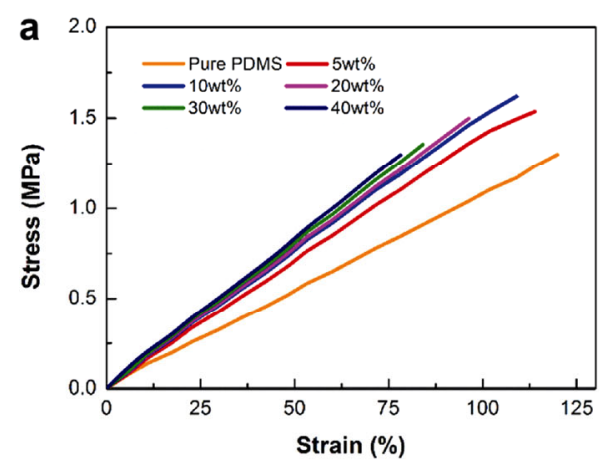

analysis XRD pattern revealed seven characteristic diffraction peaks ((100), (110), (111), (200), (210), (211), (220)). The results correspond well with the ferroelectric tetragonal phase of $\mathrm{BaTiO}_{3}$ (JCPDS No. 31-0174) and confirmed the amorphous phase of the PDMS.

The mechanical performance is critical to the flexible/ wearable devices. The relationship between the strain and stress was studied and shown in Fig. 3(a). It can be observed that before adding $\mathrm{BaTiO}_{3}$, the pure PDMS has a lower strength but a higher elongation at break. After adding $\mathrm{BaTiO}_{3}$, the strength becomes decreasing and a lower elongation at break. The results show a good agreement with other reported works [50,51]. The relationship between the filler concentration and fracture strength was studied and shown in Fig. 3(b). It can be found that the fracture strength decreases with adding the BT filler. The decrease in mechanical properties is caused by the poor matrix-filler interaction between the PDMS and $\mathrm{BaTiO}_{3}$ particles.

\section{2 Dielectric properties}

The plots of dielectric constant and dielectric loss against frequency for different concentrations of BT/PDMS composite films are displayed in Figs. 4(a) and 4(b), respectively. The size of this BT particle is about $50-80 \mathrm{~nm}$. It was found that the dielectric constant was increasing with the concentration of BT because of the introduction of inorganic fillers with high dielectric constant $[52,53]$. In addition, the dielectric constant of BT/PDMS composite decreases first at a low frequency $(<600 \mathrm{~Hz})$ and then becomes steady. The possible reason can be explained by the relatively high loss value at the low frequency range and the similar trend. Figures 4(c) and 4(d) demonstrate the comparison of frequency dependence of the dielectric constant and loss of composites with

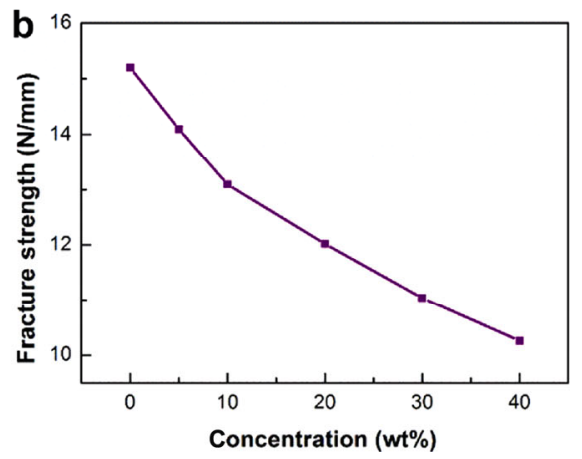

Fig. 3 (a) Stress-strain curve of the pure PDMS and the BT/PDMS nanocomposite films with different BT concentration. (b) Fracture strength of the BT/PDMS nanocomposite films with different BT concentration. 

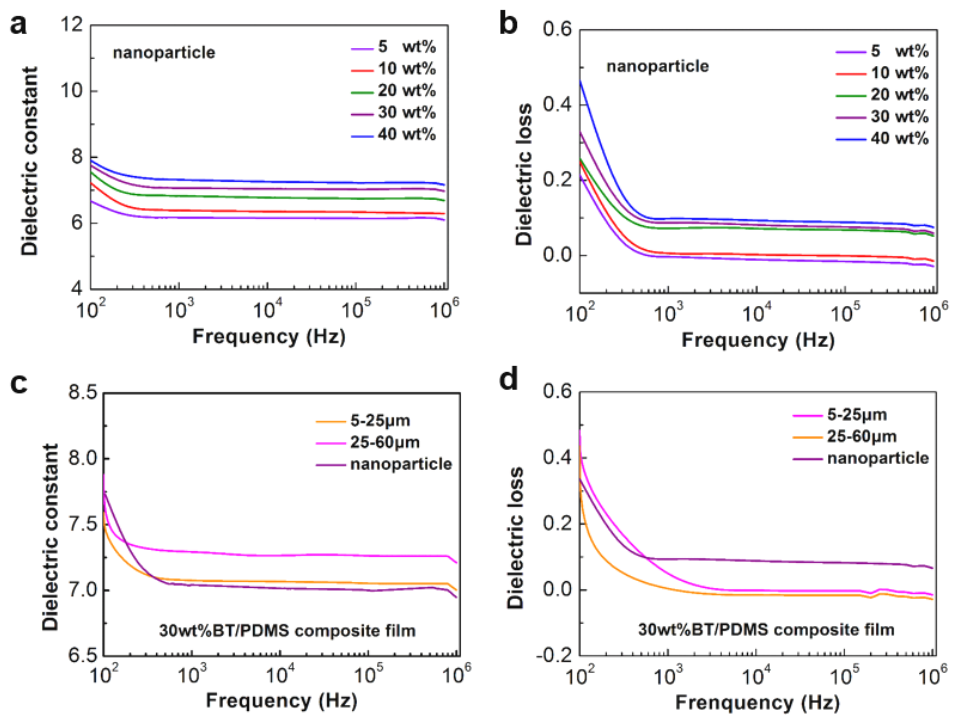

Fig. 4 Frequency dependence of (a) dielectric constant and (b) dielectric loss of BT/PDMS composite films with different concentrations of nano-sized particles. Frequency dependence of (c) dielectric constant and (d) dielectric loss of BT/PDMS composite films with $30 \mathrm{wt} \%$ of particles: 5-25 $\mu \mathrm{m}, 25-60 \mu \mathrm{m}$, and 50-80 $\mathrm{nm}$.

$30 \mathrm{wt} \%$ of fillers with different filler sizes: $5-25 \mu \mathrm{m}$, 25-60 $\mu \mathrm{m}$, and 50-80 $\mathrm{nm}$. As shown in the result, the dielectric constant increases with the size of BT filler and a tiny decrease with frequency. The composite with nano-sized filler exhibits lower dielectric constant and higher loss from $1 \mathrm{kHz}$ to high frequency range, which is like the previous report in polymer-based composites [54].

The temperature dependence of dielectric constant and loss of BT/PDMS composite films are shown in Figs. 5(a)-5(d). The details of the concentration of BT and frequency are labeled in the figures. When the temperature was rising, the dielectric constant was decreasing slightly, and the dielectric loss was approximate steady. From the value of the dielectric
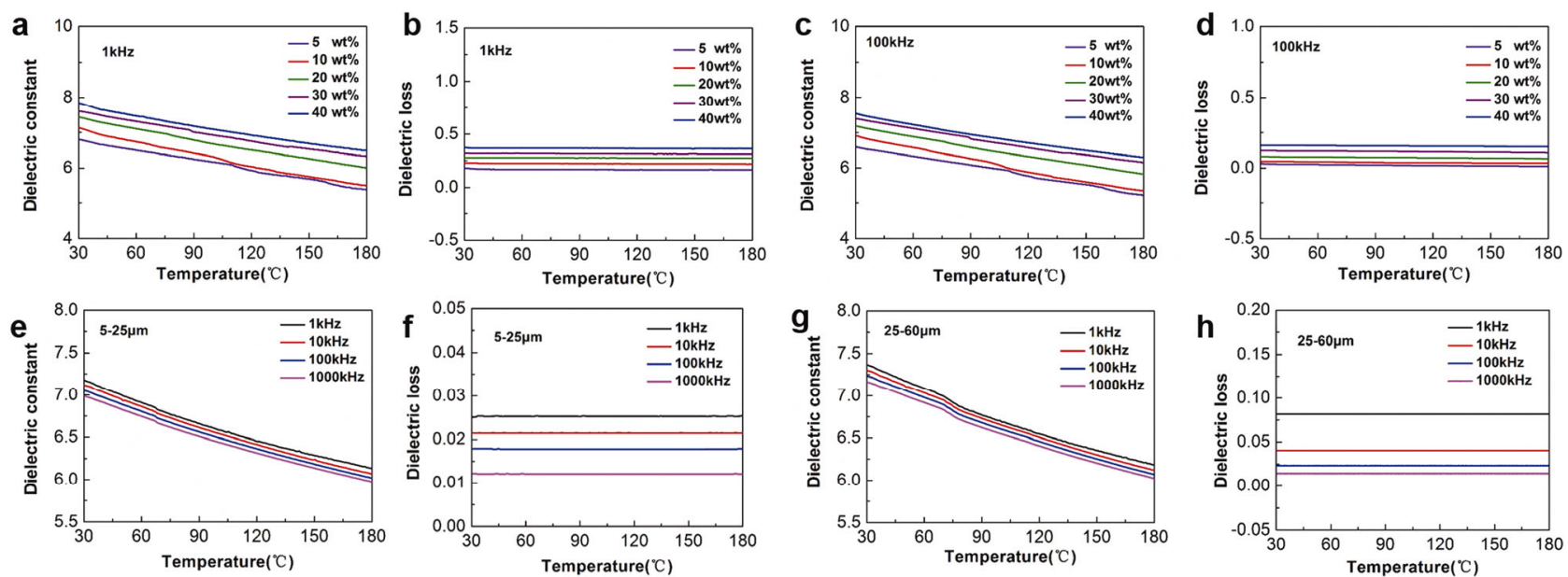

Fig. 5 Temperature dependence of dielectric constant and dielectric loss with five nanoparticle BT/PDMS composite films at four different frequencies: (a, b) $1 \mathrm{kHz},(\mathrm{c}, \mathrm{d}) 100 \mathrm{kHz}$. Temperature dependence of dielectric constant and dielectric loss with micro-scale BT/PDMS composite films: (e, f) BT flake with size of 5-25 $\mu \mathrm{m},(\mathrm{g}, \mathrm{h})$ BT flake with size of $25-60 \mu \mathrm{m}$. 


\section{3 Energy harvesting performance}

Figure 6 shows the electrical output performance of the fabricated PENG and TENG devices with different concentrations of BT/PDMS composite films (5, 10, 20, $30,40 \mathrm{wt} \%$ ) and micro-scale and nano-scale BT. The output voltage and current were measured under the same situation (frequency, external force, etc.). It was observed that both output voltage and current were increased significantly with the concentration of BT nanoparticle filler. It can be explained that the dielectric constant was increasing with the concentration of BT, and a higher dielectric constant of nanocomposite films has a good capability of storage charge, which can lead to a better performance of nanogenerators. It was demonstrated that the output voltage and current of PENG with $40 \mathrm{wt} \%$ BT were nearly three times higher than PENG with $5 \mathrm{wt} \% \mathrm{BT}$. The average output voltage and current of PENG with $40 \mathrm{wt} \%$ BT reached about $18 \mathrm{~V}$ and $0.24 \mu \mathrm{A}$, respectively. Similar results can be found on the electrical output performance of TENG in Figs. 6(d) and 6(e). The output performance of TENG is much more excellent than PENG which has been studied widely. From Figs. 6(a) and 6(d), the output voltage of TENG is ten times than PENG. The output voltage and current of $40 \mathrm{wt} \%$ BT/PDMS TENG reach $200 \mathrm{~V}$ and $0.4 \mu \mathrm{A}$, respectively. For the different sizes of BT, it can be observed that the output performance of nanogenerators with nano-scale is enhancing significantly than micro-scale. The reason is that the electrical performance is associated with the resistance of material, and the resistance of material is governed by the sintering temperature. Generally, a higher sintering temperature can lead to an increase in resistance of material. The micro-scale flakes need a higher sintering temperature than nano-scale. Therefore, the micro-scale flakes have a relatively high resistance than nano-scale. The electric output performance of nanogenerators with nano-scale is better than microscale. On the other hand, the shape and distribution of the micro-scale flakes are various and random, and the shape and size of nanoparticles are almost the same and show good arrangement. Figures 6(c) and 6(f) show the compared results of the output performance of PENG and TENG with different concentrations and sizes of BT, respectively. For the various concentrations of BT nanoparticles, the output voltage and current are increasing with the concentration. The output performance of devices based on different sizes of BT composite films was compared, too. The PENG-based micro-scale BT nanocomposite film shows better performance than the nano-scale BT under the same concentration. More specifically, the output performance of PENG-based nano-scale BT nanocomposite film enhanced approximately $30 \%$ than micro-scale $\mathrm{BT}$ nanocomposite film. The same trend results of TENG are shown in Fig. 6(f). It can also be confirmed that the output performance of TENG is better than PENG. Figure 6(g) shows the comparison of the output performance of NGs operating with the piezoelectric and triboelectric. It can be found that both the output current and voltage of triboelectric nanogenerator are much higher than piezoelectric nanogenerator. More specifically, the output performance of TENG is two times than that of PENG. Figure 6(h) shows the output power of $40 \mathrm{wt} \%$ BT/PDMS-based PENG and TENG with the external load resistance ranging from $10^{2}$ to $10^{9} \Omega$. The peak output power of TENG reaches up to $0.16 \mathrm{~mW}$ and it can be employed in many applications. In addition, as shown in Fig. 6(i), the output performance of nanogenerators based on BT/PDMS particles is still stable after 1000 cycles.

Figure 7(a) shows the working principle of TENG. At the initial position, there are no charges on the two surfaces and no potential difference between the two electrodes. With an external physical force pressing on the device, the two layers are going to access and contact with each other. At this time, the opposite charge is occurred on the two surfaces because of the triboelectric effect the opposite charges have occurred on the electrodes. The electrons in one electrode would transfer to the other electrode to balance the electrostatic field. When the TENG is released to the origin state, the output voltage will keep raising to the maximum value. The cycled contact and separation process of the two contact layers drives the induced electrons in the circuit to flow back and forth to build an AC output. Compared to PENG, the piezoelectric-based TENG combines both piezoelectric and triboelectric effect together, leading to an enhanced output performance. The studies shown in Fig. 6 provide several understandings on the piezoelectric filler/PMDS-based PENG/TENG. First, the output performance of both devices enhances with the increase of the content of piezoelectric filler because more piezoelectric fillers are in the matrix which provide more charge during the pressing/releasing. Second, the micro-sized filler shows lower output power then nano-sized filler when the devices have the 

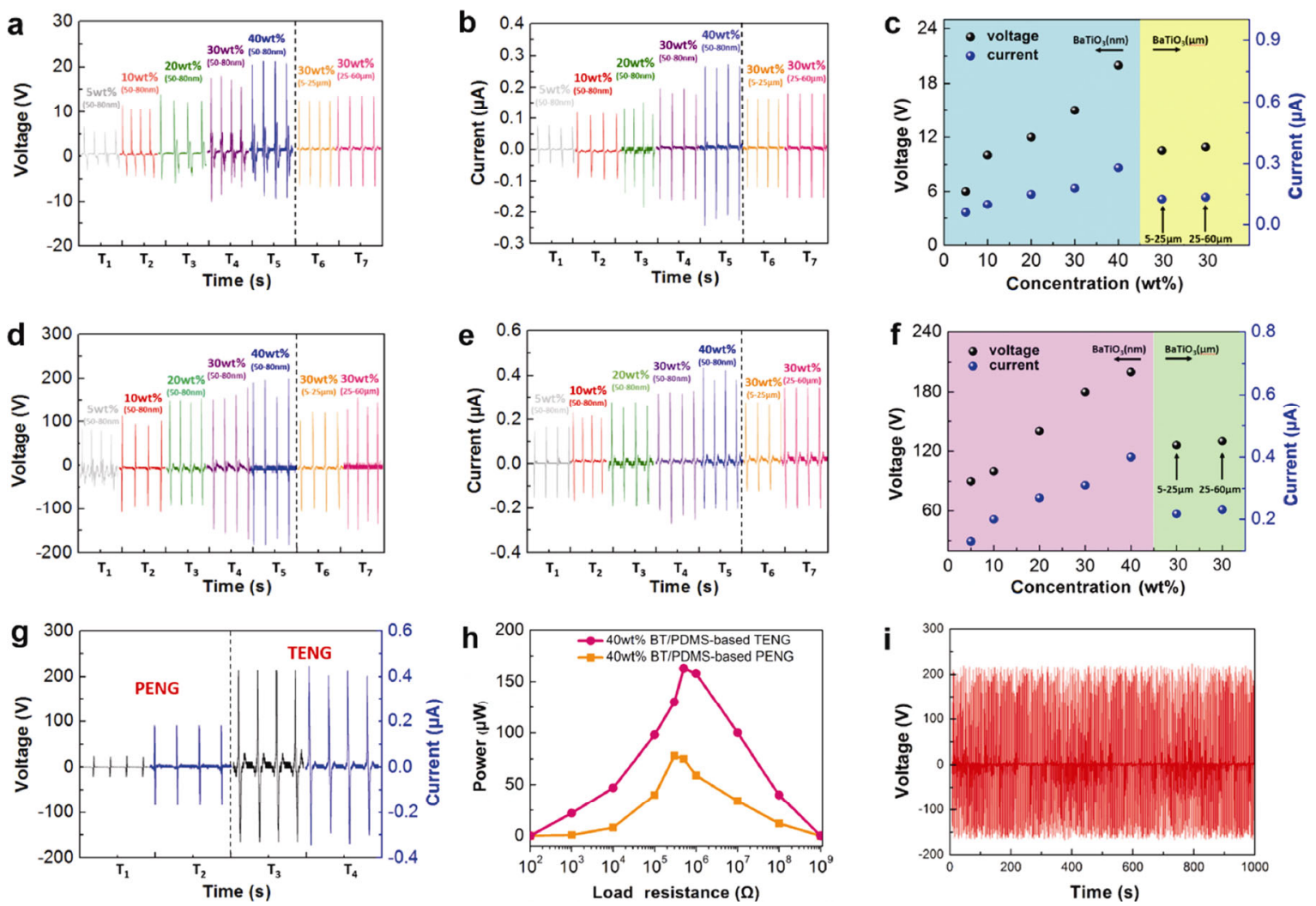

Fig. 6 Output performance of PENG and TENG with different concentrations and sizes of BT. The output voltage (a) and current (b) of PENG, and (c) the comparison of the output performance of PENG with different concentrations and sizes of BT. The output voltage (d) and current (e) of TENG, and (f) the comparison of the output performance of TENG with different concentrations and sizes of BT. (g) Comparison of the output performance of PENG and TENG. (h) Output power of $40 \mathrm{wt} \%$ BT/PDMS-based PENG and TENG devices with the external load resistance ranging from $10^{2}$ to $10^{9} \Omega$. (i) Durability test of 40 $\mathrm{wt} \% \mathrm{BT} / \mathrm{PDMS}-$ based TENG. The rate is $1 \mathrm{cycle} / \mathrm{s}$.
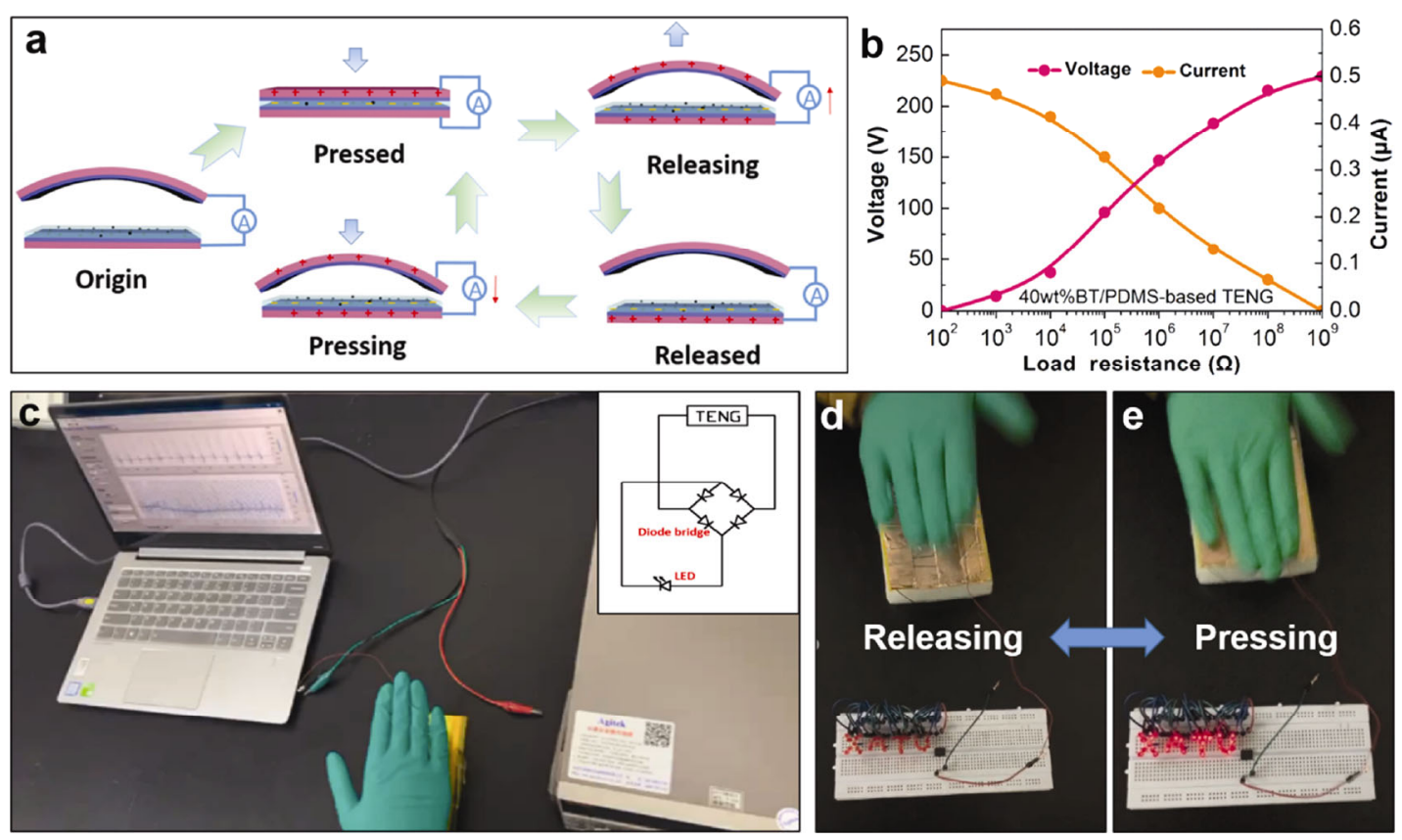

Fig. 7 (a) Schematic depicting the working principle of TENG. (b) Voltage/current of $40 \mathrm{wt} \%$ BT/PDMS-based TENG device with the external load resistance ranging from $10^{2}$ to $10^{9} \Omega$. (c) The image of the measurement system and the schematic diagram of a diode bridge. (d, e) The photographs of commercial LEDs connected in series directly lightened by of TENG during the pressing/releasing process. 
same concentration. The reason may raise from two aspects: i) the large distribution of size and shape of the BT flakes may result in an inhomogeneous microstructure and non-uniform charge distribution, ii) the same weight percent leads to the less particles of BT flakes in the matrix compared to the nanoparticles, and iii) the possible flakes are broken during the pressing/releasing process. Third, it clearly shows that the TENG exhibits higher value of both output current and voltage compared to the PENG, which can be ascribed to the synergetic effect of both the piezoelectric and triboelectric effects: i) the triboelectric friction between two layers could generate positive and negative charges across their surfaces, and ii) the compression on the piezoelectric composites generates the piezoelectric potential caused by the resultant electric dipole moments.

For practical applications, the effect of external load resistance on the output performance of the TENG device was also tested and the result is showed in Fig. 7(b). As the external load was increasing from $10^{4}$ to $10^{8} \Omega$, the peak-peak voltage increased significantly. Figure $7(\mathrm{c})$ shows the measurement system, which consists of an electrometer and a computer. The schematic diagram of a full rectifying diode bridge is attached on the top right corner. It can be connected to the load to convert AC to DC to use to charge energy storage devices such as batteries or supercapacitors. Figures 7(d) and 7(e) exhibit the photographic images of a logo of XATU, which made of many commercial light-emitting diodes (LEDs). The LEDs are brightened instantly by the power generated from the TENG through the rectifier when the force was releasing and pressing, respectively. The results indicate that the BT/PDMS TENG device can be utilized as a selfpowered source for wearable/portable electronics.

\section{Conclusions}

The BT/PDMS composite films are systematically investigated and utilized for energy harvesting applications. The composites were fabricated by the spincoating method with different concentrations of nanosized BT particles from 5 to $40 \mathrm{wt} \%$ and micro-sized BT flakes. The dependence of dielectric constant and dielectric loss of the BT/PDMS composite films on frequency and temperature was investigated. It is found that the dielectric constant and loss were increasing significantly with the concentration of the nanoparticles and decreasing with the frequency. The value of the dielectric loss of $40 \mathrm{wt} \% \mathrm{BT} / \mathrm{PDMS}$ was still in a low value $(\sim 0.12)$. The dielectric constant was influenced by temperature and the dielectric loss was almost steady with the temperature. The output performance of PENG and TENG is enhancing by the concentration of BT/PDMS composite films. The output voltage and current of $40 \mathrm{wt} \%$ BT/PDMS TENG reach $200 \mathrm{~V}$ and $0.4 \mu \mathrm{A}$, respectively, which are more than two times higher than that with $5 \mathrm{wt} \%$ of BT. It clearly shows that the TENG exhibits higher value of both output current and voltage compared to the PENG, which can be ascribed to the synergetic effect of both the piezoelectric and triboelectric effects. The stable and high electrical output power generated by the TENG was utilized to light commercial LEDs, which suggests that the device has the potential to the energy harvesting in biomedical application.

\section{Acknowledgements}

The work was supported by the National Natural Science Foundation of China (Grant No. 51972263).

\section{References}

[1] Von Buren T, Mitcheson PD, Green TC, et al. Optimization of inertial micropower Generators for human walking motion. IEEE Sensor J 2006, 6: 28-38.

[2] Bai P, Zhu G, Lin ZH, et al. Integrated multilayered triboelectric nanogenerator for harvesting biomechanical energy from human motions. ACS Nano 2013, 7: 3713-3719.

[3] Yang WQ, Chen J, Zhu G, et al. Harvesting energy from the natural vibration of human walking. ACS Nano 2013, 7: 11317-11324.

[4] Sari I, Balkan T, Kulah H. An electromagnetic micro power generator for wideband environmental vibrations. Sensor Actuat A: Phys 2008, 145-146: 405-413.

[5] Yang WQ, Chen J, Jing QS, et al. 3D stack integrated triboelectric nanogenerator for harvesting vibration energy. Adv Funct Mater 2014, 24: 4090-4096.

[6] Xie YN, Wang SH, Lin L, et al. Rotary triboelectric nanogenerator based on a hybridized mechanism for harvesting wind energy. ACS Nano 2013, 7: 7119-7125.

[7] $\mathrm{Su} \mathrm{YJ,} \mathrm{Wen} \mathrm{XN,} \mathrm{Zhu} \mathrm{G,} \mathrm{et} \mathrm{al.} \mathrm{Hybrid} \mathrm{triboelectric}$ nanogenerator for harvesting water wave energy and as a self-powered distress signal emitter. Nano Energy 2014, 9: 186-195.

[8] Parvez AN, Rahaman MH, Kim HC, et al. Optimization of triboelectric energy harvesting from falling water droplet 
onto wrinkled polydimethylsiloxane-reduced graphene oxide nanocomposite surface. Compos Part B: Eng 2019, 174: 106923.

[9] Fan X, Chen J, Yang J, et al. Ultrathin, rollable, paperbased triboelectric nanogenerator for acoustic energy harvesting and self-powered sound recording. ACS Nano 2015, 9: 4236-4243.

[10] Fan FR, Tian ZQ, Zhong LW. Flexible triboelectric generator. Nano Energy 2012, 1: 328-334.

[11] Zhang KW, Zhang L, Fu LL, et al. Magnetostrictive resonators as sensors and actuators. Sensor Actuat A: Phys 2013, 200: 2-10.

[12] Dagdeviren C, Joe P, Tuzman OL, et al. Recent progress in flexible and stretchable piezoelectric devices for mechanical energy harvesting, sensing and actuation. Extrem Mech Lett 2016, 9: 269-281.

[13] Miao P, Mitcheson PD, Holmes AS, et al. MEMS inertial power generators for biomedical applications. Microsyst Technol 2006, 12: 1079-1083.

[14] Narita F, Fox M. A review on piezoelectric, magnetostrictive, and magnetoelectric materials and device technologies for energy harvesting applications. Adv Eng Mater 2018, 20: 1700743.

[15] Wang ZL, Song J. Piezoelectric nanogenerators based on zinc oxide nanowire arrays. Science 2006, 312: 242-246.

[16] Wang ZL. Triboelectric nanogenerators as new energy technology and self-powered sensors-Principles, problems and perspectives. Faraday Discuss 2014, 176: 447-458.

[17] Niu SM, Wang SH, Lin L, et al. Theoretical study of contact-mode triboelectric nanogenerators as an effective power source. Energy Environ Sci 2013, 6: 3576.

[18] Wang SH, Lin L, Xie YN, et al. Sliding-triboelectric nanogenerators based on in-plane charge-separation mechanism. Nano Lett 2013, 13: 2226-2233.

[19] Yang Y, Zhou YS, Zhang HL, et al. A single-electrode based triboelectric nanogenerator as self-powered tracking system. Adv Mater 2013, 25: 6594-6601.

[20] Wang SH, Xie YN, Niu SM, et al. Freestanding triboelectriclayer-based nanogenerators for harvesting energy from a moving object or human motion in contact and non-contact modes. Adv Mater 2014, 26: 2818-2824.

[21] Song YH, Shi ZQ, Hu GH, et al. Recent advances in cellulose-based piezoelectric and triboelectric nanogenerators for energy harvesting: A review. J Mater Chem A 2021, 9: 1910-1937.

[22] Maiti S, Karan SK, Kim JK, et al. Nature driven biopiezoelectric/triboelectric nanogenerator as next-generation green energy harvester for smart and pollution free society. Adv Energy Mater 2019, 9: 1803027.

[23] Chen XX, Ren ZY, Han MD, et al. Hybrid energy cells based on triboelectric nanogenerator: From principle to system. Nano Energy 2020, 75: 104980.

[24] Chen C, Bai ZK, Cao YZ, et al. Enhanced piezoelectric performance of $\mathrm{BiCl}_{3} / \mathrm{PVDF}$ nanofibers-based nanogenerators. Compos Sci Technol 2020, 192: 108100.
[25] Hu PH, Yan LL, Zhao CX, et al. Double-layer structured PVDF nanocomposite film designed for flexible nanogenerator exhibiting enhanced piezoelectric output and mechanical property. Compos Sci Technol 2018, 168: 327-335.

[26] Ko YH, Nagaraju G, Lee SH, et al. PDMS-based triboelectric and transparent nanogenerators with $\mathrm{ZnO}$ nanorod arrays. ACS Appl Mater Interfaces 2014, 6: 6631-6637.

[27] Jian G, Meng QZ, Jiao Y, et al. Enhanced performances of triboelectric nanogenerators by filling hierarchical flower-like $\mathrm{TiO}_{2}$ particles into polymethyl methacrylate film. Nanoscale 2020, 12: 14160-14170.

[28] Lee DW, Jeong DG, Kim JH, et al. Polarization-controlled PVDF-based hybrid nanogenerator for an effective vibrational energy harvesting from human foot. Nano Energy 2020, 76: 105066.

[29] Zhou ZJ, Li JL, Xia WM, et al. Enhanced piezoelectric and acoustic performances of poly(vinylidene fluoridetrifluoroethylene) films for hydroacoustic applications. Phys Chem Chem Phys 2020, 22: 5711-5722.

[30] Lu X, Hou L, Zhang L, et al. Piezoelectric-excited membrane for liquids viscosity and mass density measurement. Sensor Actuat A: Phys 2017, 261: 196-201.

[31] Abolhasani MM, Shirvanimoghaddam K, Naebe M. $\mathrm{PVDF} /$ graphene composite nanofibers with enhanced piezoelectric performance for development of robust nanogenerators. Compos Sci Technol 2017, 138: 49-56.

[32] Park H, Hyeon DY, Jung M, et al. Piezoelectric $\mathrm{BaTiO}_{3}$ microclusters and embossed $\mathrm{ZnSnO}_{3}$ microspheres-based monolayer for highly-efficient and flexible composite generator. Compos Part B: Eng 2020, 203: 108476.

[33] $\mathrm{He} \mathrm{XM}, \mathrm{Mu} \mathrm{XJ}$, Wen Q, et al. Flexible and transparent triboelectric nanogenerator based on high performance well-ordered porous PDMS dielectric film. Nano Res 2016, 9: 3714-3724.

[34] Wang C, Li X, Hu H, et al. Monitoring of the central blood pressure waveform via a conformal ultrasonic device. Nat Biomed Eng 2018, 2: 687-695.

[35] Hu H, Zhu X, Wang C, et al. Stretchable ultrasonic transducer arrays for three-dimensional imaging on complex surfaces. Sci Adv 2018, 4: eaar3979.

[36] Briscoe J, Dunn S. Piezoelectric nanogenerators - a review of nanostructured piezoelectric energy harvesters. Nano Energy 2015, 14: 15-29.

[37] Jiang W, Li H, Liu Z, et al. Fully bioabsorbable natural-materials-based triboelectric nanogenerators. $A d v$ Mater 2018, 30: e1801895.

[38] Park KI, Son JH, Hwang GT, et al. Highly-efficient, flexible piezoelectric PZT thin film nanogenerator on plastic substrates. Adv Mater 2014, 26: 2514-2520.

[39] Sun Y, Liu Y, Zheng YD, et al. Enhanced energy harvesting ability of $\mathrm{ZnO} / \mathrm{PAN}$ hybrid piezoelectric nanogenerators. ACS Appl Mater Interfaces 2020, 12: 54936-54945.

[40] Le AT, Ahmadipour M, Pung SY. A review on ZnO-based piezoelectric nanogenerators: Synthesis, characterization 
techniques, performance enhancement and applications. $J$ Alloys Compd 2020, 844: 156172.

[41] Zhuang YY, Li JL, Hu QY, et al. Flexible composites with $\mathrm{Ce}$-doped $\mathrm{BaTiO}_{3} / \mathrm{P}(\mathrm{VDF}-\mathrm{TrFE})$ nanofibers for piezoelectric device. Compos Sci Technol 2020, 200: 108386.

[42] Patnam H, Dudem B, Alluri NR, et al. Piezo/triboelectric hybrid nanogenerators based on Ca-doped Barium zirconate titanate embedded composite polymers for wearable electronics. Compos Sci Technol 2020, 188: 107963.

[43] Lu X, Zou XW, Shen JL, et al. Characterizations of $\mathrm{P}\left(\right.$ VDF-HFP)- $\mathrm{BaTiO}_{3}$ nanocomposite films fabricated by a spin-coating process. Ceram Int 2019, 45: 17758-17766.

[44] Wen F, Lou HY, Ye JF, et al. Preparation and energy storage performance of transparent dielectric films with twodimensional platelets. Compos Sci Technol 2019, 182: 107759.

[45] Shi KM, Huang XY, Sun B, et al. Cellulose/ $\mathrm{BaTiO}_{3}$ aerogel paper based flexible piezoelectric nanogenerators and the electric coupling with triboelectricity. Nano Energy 2019, 57: 450-458.

[46] Lu X, Zhang L, Talebinezhad $\mathrm{H}$, et al. Effects of $\mathrm{CuO}$ additive on the dielectric property and energy-storage performance of $\mathrm{BaTiO}_{3}-\mathrm{SiO}_{2}$ ceramic-glass composite. Ceram Int 2018, 44: 16977-16983.

[47] Guo HL, Wu Q, Sun HJ, et al. Organic phosphonic acid-modified $\mathrm{BaTiO}_{3} / \mathrm{P}(\mathrm{VDF}-\mathrm{TrFE})$ composite with high output in both voltage and power for flexible piezoelectric nanogenerators. Mater Today Energy 2020, 17: 100489.

[48] $\mathrm{Su} \mathrm{HX}$, Wang $\mathrm{XB}$, Li CY, et al. Enhanced energy harvesting ability of polydimethylsiloxane- $\mathrm{BaTiO}_{3}$-based flexible piezoelectric nanogenerator for tactile imitation application. Nano Energy 2021, 83: 105809.

[49] Sriphan S, Nawanil C, Vittayakorn N. Influence of dispersed phase morphology on electrical and fatigue properties of $\mathrm{BaTiO}_{3} / \mathrm{PDMS}$ nanogenerator. Ceram Int 2018, 44: S38-S42.

[50] Tang ZH, Gao ZW, Jia SH, et al. Graphene-based polymer bilayers with superior light-driven properties for remote construction of 3D structures. Adv Sci 2017, 4: 1600437.

[51] Kausar A. Polydimethylsiloxane-based nanocomposite: Present research scenario and emergent future trends. Polym - Plast Technol Mater 2020, 59: 1148-1166.

[52] Zhang L, Wu PX, Li YT, et al. Preparation process and dielectric properties of $\mathrm{Ba}_{0.5} \mathrm{Sr}_{0.5} \mathrm{TiO}_{3}-\mathrm{P}$ (VDF-CTFE) nanocomposites. Compos Part B: Eng 2014, 56: 284-289.

[53] Zhang L, Zhang L, Shan X, et al. Process and microstructure to achieve ultra-high dielectric constant in ceramic-polymer composites. Sci Rep 2016, 6: 35763.

[54] Shan XB, Zhang L, Yang XQ, et al. Dielectric composites with a high and temperature-independent dielectric constant. J Adv Ceram 2012, 1: 310-316.

Open Access This article is licensed under a Creative Commons Attribution 4.0 International License, which permits use, sharing, adaptation, distribution and reproduction in any medium or format, as long as you give appropriate credit to the original author(s) and the source, provide a link to the Creative Commons licence, and indicate if changes were made.

The images or other third party material in this article are included in the article's Creative Commons licence, unless indicated otherwise in a credit line to the material. If material is not included in the article's Creative Commons licence and your intended use is not permitted by statutory regulation or exceeds the permitted use, you will need to obtain permission directly from the copyright holder.

To view a copy of this licence, visit http://creativecommons.org/licenses/by/4.0/. 